\title{
Porous graphene oxide films prepared via the breath-figure method: a simple strategy for switching access of redox species to an electrode surface
}

Rumei Cheng,,$^{a^{\times}}$Rafael N. P. Colombo, ${ }^{b \times}$ Long Zhang, ${ }^{c}$ Duyen H.T. Nguyen, ${ }^{c}$ Richard Tilley, ${ }^{c, d}$ Susana I. Cordoba de Torresi, ${ }^{b}$ Liming Dai, ${ }^{e}$ J. Justin Gooding, ${ }^{c *}$ Vinicius $R$.

Gonçales, ${ }^{c *}$

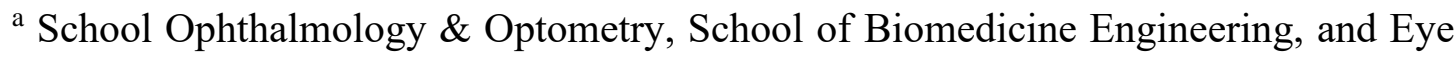
Hospital, Wenzhou Medical University, Wenzhou, Zhejiang 325027, China

b Instituto de Química, Universidade de São Paulo, Av. Prof. Lineu Prestes 748, 05508-000 São Paulo, Brazil

c School of Chemistry, Australia Centre for NanoMedicine, ARC Centre of Excellence in Convergent Bio-Nano Science and Technology, The University of New South Wales, Sydney NSW2052, Australia

${ }^{\mathrm{d}}$ Electron Microscopy Unit, Mark Wainwright Analytical Centre, The University of New South Wales, Sydney NSW2052, Australia

e School of Chemistry Engineering, The University of New South Wales, Sydney NSW2052, Australia

${ }^{\times}$Authors contributed equally to the work

E-mails: justin.gooding@unsw.edu.au; v.goncales@unsw.edu.au 


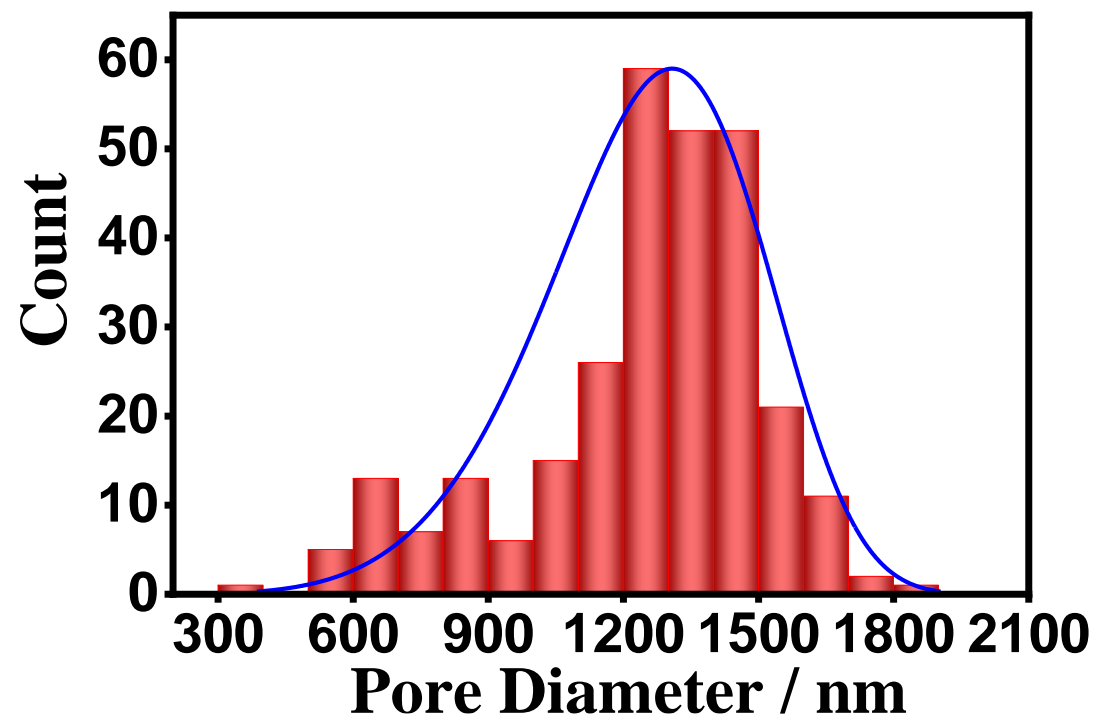

Figure S1 - Pore diameter distribution of porous GO-DODA on ITO electrodes. Weibull distribution curve represented in blue with an average pore size of $1.35 \pm$ $0.33 \mu \mathrm{m}$.

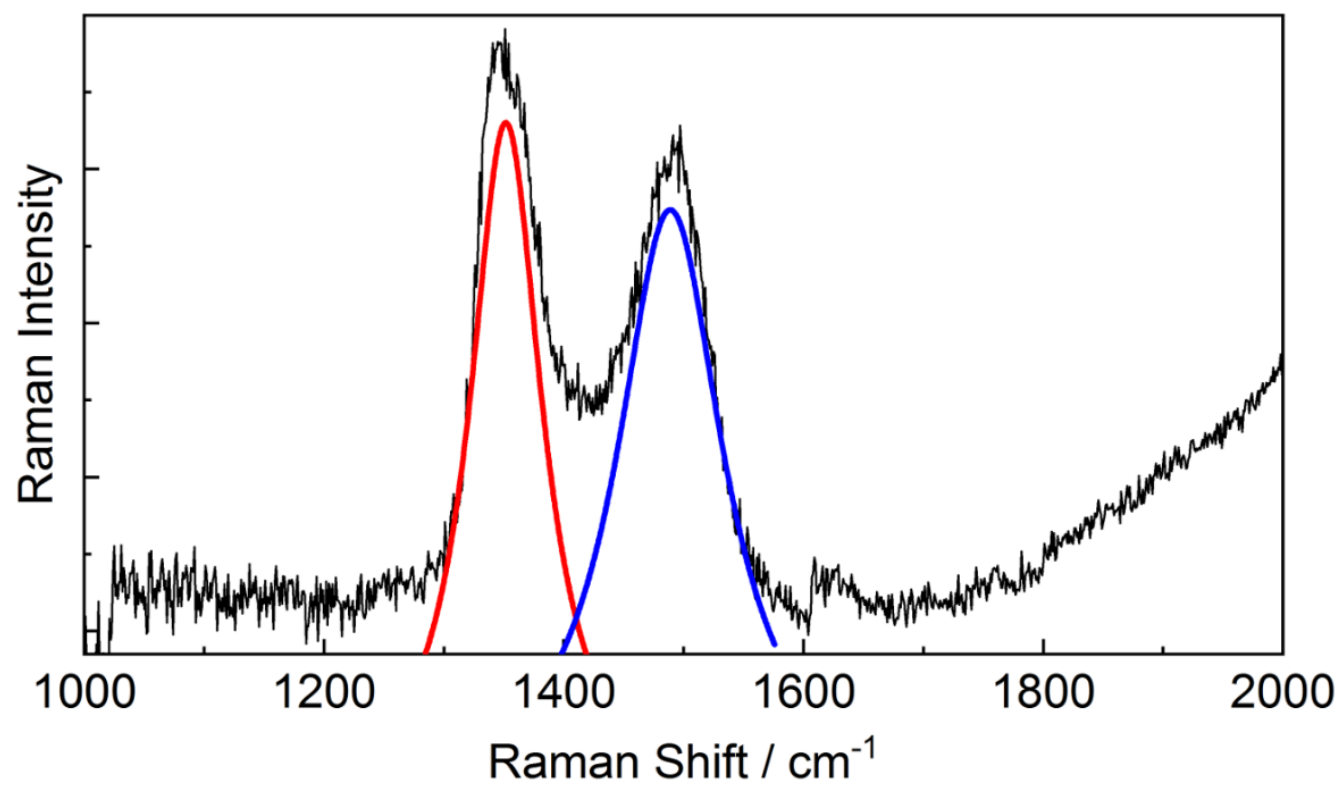

Figure S2. Raman spectrum of GO-DODA with deconvoluted Lorentzian curves for D and $\mathrm{G}$ bands (red and blue, respectively); $\mathrm{I}_{\mathrm{D}} / \mathrm{I}_{\mathrm{G}}$ ratio $=0.8$. 


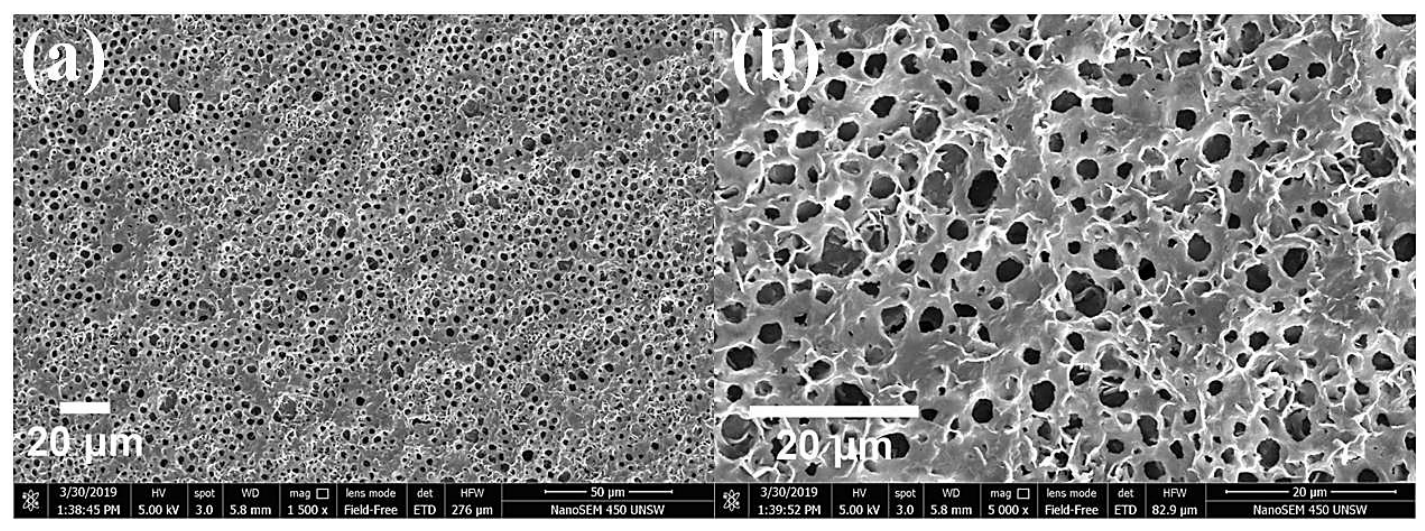

Figure S3 - SEM micrographs of an ITO|GO-DODA-DDAT surface at (a) lower and (b) higher magnifications. ITO|GO-DODA reacted with DDAT for $3 \mathrm{~h}$ in order to create anchorage points for the localised in-situ growth of thermoresponsive pNIPAM brushes, as shown in Scheme 2. 

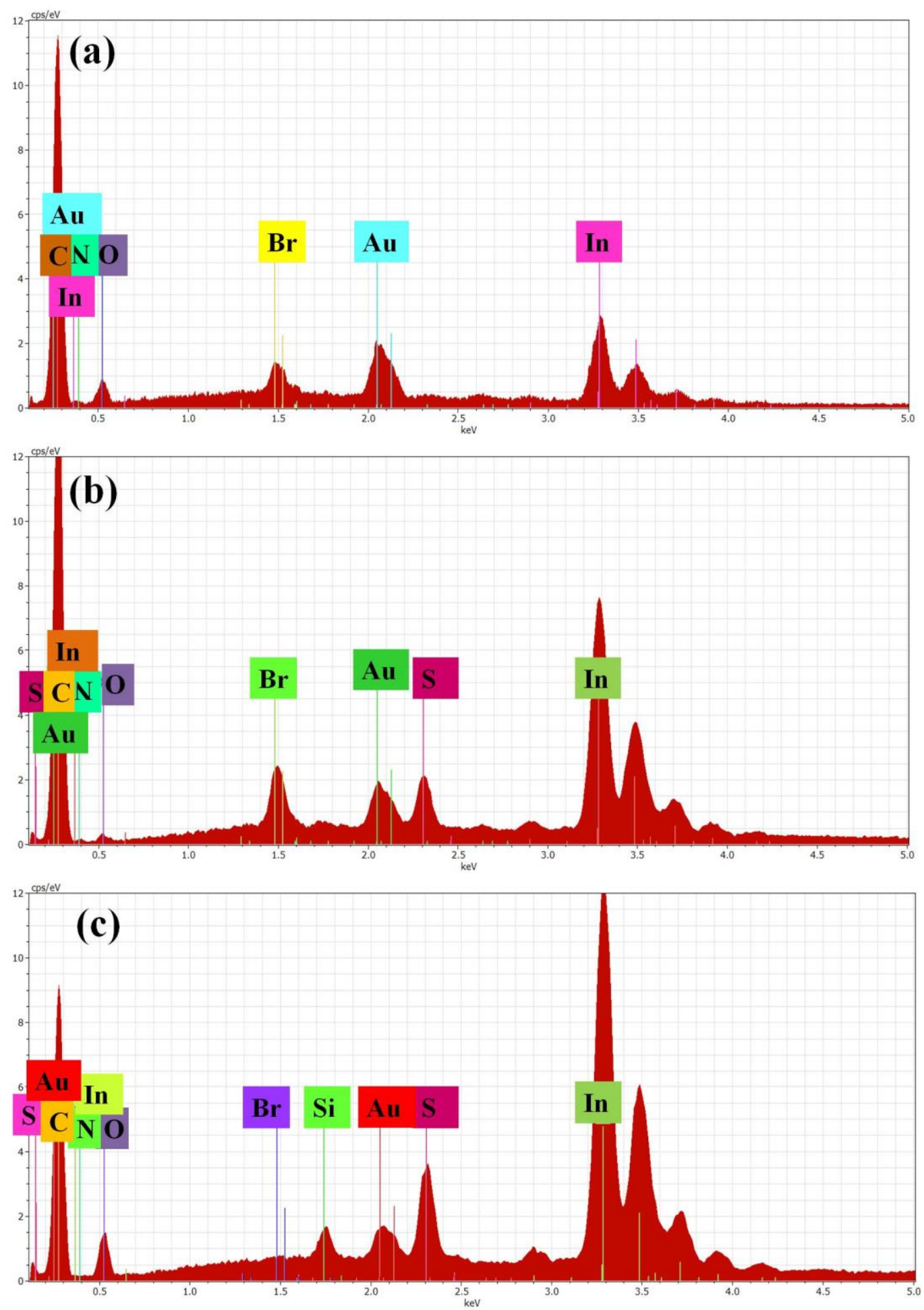

Figure S4 - EDS spectra of (a) GO-DODA, (b) GO-DODA-DDAT and (c) GO-DODA-DDAT-pNIPAM surfaces. Gold comes from the thin layer sputtered for SEM analysis. Indium is due to ITO, and $\mathrm{Br}$ is present from the DODA surfactant and washed away after DDAT and pNIPAM incorporation. 


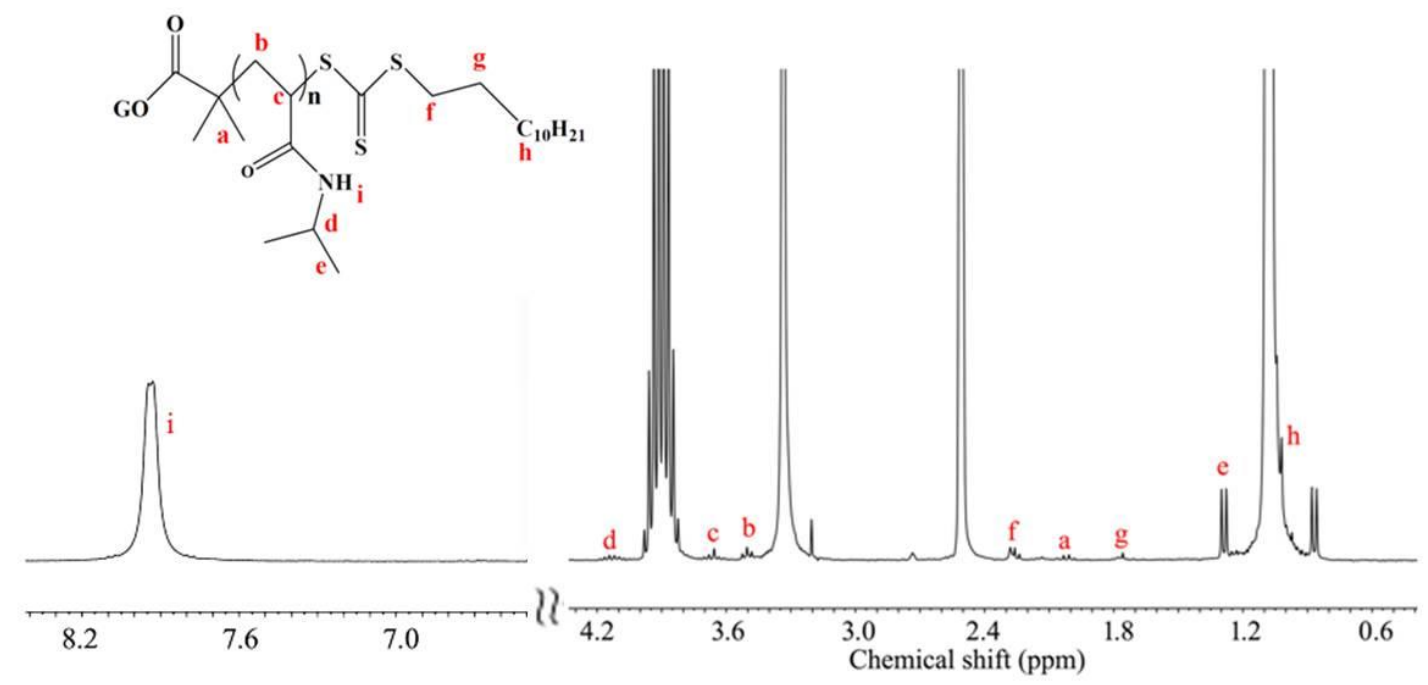

Figure S5 - ${ }^{1}$ HNMR spectrum of GO-DODA-DDAT-pNIPAM in DMSO-d 6 . A solvent spike is found at $2.52 \mathrm{ppm}$, and water at $3.2 \mathrm{ppm}$ is due to moisture absorbed from air. 

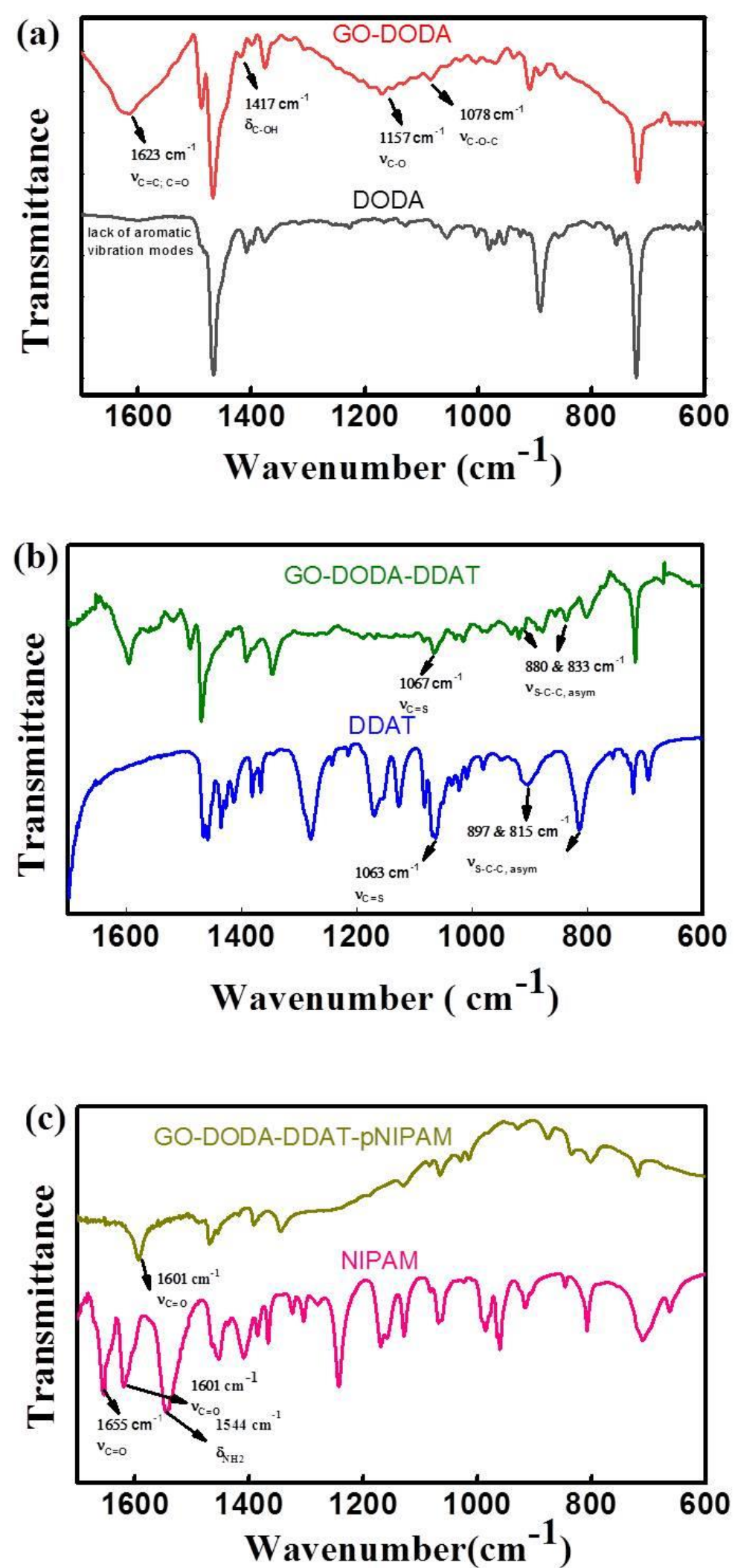

Figure S6 - (a) FTIR-ATR spectra of DODA and GO-DODA; (b) DDAT and GO-DODA-DDAT; (c) NIPAM and GO-DODA-DDAT-pNIPAM. 


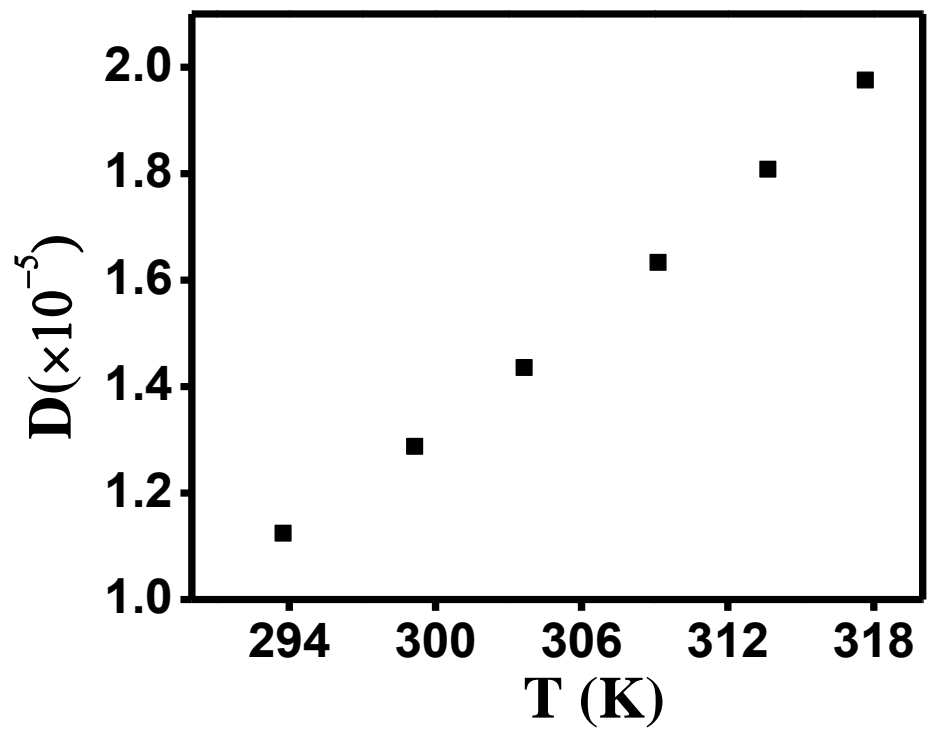

Figure S7 -Linear relationship between D and T of ITO at different temperatures. 